Research paper

\title{
Void fraction measurement in cryogenic flows. Part II: Void fraction capacitive sensor performances in chilldown experiments
}

\author{
Yuki Sakamoto ${ }^{\mathrm{a}, *, 1}$, Laura Peveroni ${ }^{\mathrm{a}}$, Hiroaki Kobayashi ${ }^{\mathrm{b}, 2}$, Tetsuya Sato ${ }^{\mathrm{c}}$, Johan Steelant ${ }^{\mathrm{d}}$, \\ Jean-Marie Buchlin ${ }^{\mathrm{a}}$ \\ a von Karman Institute, Chaussee De Waterloo 72, B-1640 Rhode-St-Genese, Belgium \\ ${ }^{\mathrm{b}}$ Japan Aerospace Exploration Agency, 7-44-1 Jindaiji Higashi-machi, Chofu, Tokyo 182-8522, Japan \\ ' Waseda University, 3-4-1 Okubo, Shinjuku-ku, Tokyo 169-8555, Japan \\ 'Aerothermodynamics and Propulsion Analysis Section, ESTEC-ESA, Keplerlaan 1, 2200AG Noordwijk, the Netherlands
}

\section{A R T I C L E I N F O}

\section{Keywords:}

Capacitive sensor

Electric field analysis

Void fraction

Chilldown

Liquid nitrogen

Cooling channel

Cryogenic

\begin{abstract}
A B S T R A C T
This manuscript describes the work performed on void fraction measurements a cryogenic flow by means of a customized capacitive sensor. In a preceding activity, described in Part I, the instrument was developed and validated at room conditions. In the current study, the probe is exploited to detect the gaseous content during liquid nitrogen chilldown experiments. The sensor performances are evaluated both numerically and experimentally. The numerical simulations lead to the development of a new calibration formula improving the sensor measurement accuracy down to $\pm 6.0 \% \mathrm{FS}$, within $99 \%$ confident interval. The experimental campaign mainly reveals a dependency of the sensor performance on the pressure and temperature variations during the cooldown of the test section. The so-called "thermal effect" therefore modeled and two compensation equations are derived. The void fraction results accordingly corrected, match the single-phase flows reference conditions within $\pm 2 \%$ discrepancy. Background light visualizations are also performed allowing the optical verification of the flow regimes. For a specific flow condition, a correlation between the recorded light intensity and the capacitive measurements is obtained. By means of the high-speed movies, the capacitive sensor response time is also evaluated to be $100 \mathrm{~Hz}$.
\end{abstract}

\section{Introduction}

The void fraction commonly intended as the fraction of a pipe volume that is occupied by the gas phase, is one of the most important parameters required to characterize a two-phase flow. As addressed in [1-3] several void fraction measurement techniques have been and are currently investigated. Various measuring principles are exploited, for example optical fibers [4], wire meshes [5], impedance changes [6] or wave propagations (such as x-rays [7] or ultrasounds [8]). However, especially for the very challenging environment and complex phenomenon related to a cryogenic chilldown, there is a lack of well-established and validated measurement methods.

In the present study, a capacitance-based measurement technique for cryogenic fluid is developed. In Part I [1], the void fraction sensor was numerically and experimentally characterized by means of a bubbly flow obtained injecting air in Polydimethylsiloxane at room temperature. Although such a configuration differs from the design application of the sensor (i.e. liquid nitrogen two-phase flow), comparing the capacitive results against optical measurements and the numerical simulations (Electric Field Analysis - EFA) it was concluded that the sensor measures the void fraction within $\pm 7 \% \mathrm{FS}$ error, for void fraction values up to $38 \%$.

The activity presented in this paper focuses then on the assessment of the capacitive instrument performance in cryogenic conditions. The CHIlldown Experimental Facility (CHIEF) at the von Karman Institute (VKI) is exploited to perform the test campaign [3,9]. High-speed camera visualizations are also conducted to support the investigation of the sensor capabilities as much as the EFA numerical simulations.

\section{Experimental facility}

The configuration of the VKI CHIEF, the instrument locations and

\footnotetext{
* Corresponding author.

E-mail address: sakamoto-yuki@ruri.waseda.jp (Y. Sakamoto).

${ }^{1}$ Current address: Waseda University, 3-4-1 Okubo, Shinjuku-ku, Tokyo 169-8555, Japan.

${ }^{2}$ Current address: Japan Aerospace Exploration Agency, 3-1-1 Yoshinodai, Chuo-ku, Sagamihara, Kanagawa 252-5210, Japan.
} 


\begin{tabular}{ll}
\multicolumn{2}{l}{ Nomenclature } \\
Subscripts \\
EFA & Electric Field Analysis \\
C & capacitance \\
CHIEF & Chilldown Experimental Facility \\
C. $I$. & Confidence Interval \\
FFT & Fast Fourier Transformation \\
HSC & High Speed Camera \\
H. S. & High Subcooling \\
L. I. & Light intensity \\
LN2 & Liquid Nitrogen \\
$M . S$. & Moderate Subcooling \\
$P$ & pressure \\
$Q$ & mass flow rate \\
$t$ & time \\
$T$ & temperature \\
VKI & Von Karman Institute
\end{tabular}

\section{Subscripts}

$\begin{array}{ll}G & \text { gas } \\ L & \text { liquid } \\ \text { linear } & \text { calculated by Eq. (1) } \\ M & \text { measured } \\ r e f & \text { Reference or Calibration value } \\ T C & \text { temperature contraction }\end{array}$

Greek symbols

$\alpha \quad$ void fraction

$\varepsilon \quad$ dielectric constant or relative permittivity

$\mu \quad$ mean value

$\sigma \quad$ standard deviation

Others

$\pm \%$ FS absolute error

$\pm \%$ rdg relative error to reading their nomenclature for the present test campaign are recalled in Fig. 1. The preliminary phase of each experiment is dedicated to the chilldown of the upstream elements by means of the by-pass line. In such a way, the inlet of the test section is provided with single-phase liquid nitrogen. The distribution valve is indeed opened to the test section only when the proper upstream pressure and temperature are reached $[3,9,10]$.

Kulites cryogenic pressure sensors (CTL-190), connected to a Fylde Micro Analog 2 conditioner, are employed to measure the absolute pressure. The wall temperature is obtained by means of LakeShore Silicon Diodes transducers (DT-670B-SD) cabled to a Temperature Monitor T218Ss. The sensors are embedded in the pipe thickness, at $1 \mathrm{~mm}$ from the inner wall surface. Finally, the Yokogawa Coriolis cryogenic mass flow meter (RCCS34) is provided also of a PT100 probe, used to monitor the fluid inlet temperature. The accuracy related to each different sensor is estimated from the datasheet and supplier calibrations (Table 1). Concerning the pressure transducers, the main source of error is due to the thermal compensation module implemented in the Wheatstone bridge circuit of the sensible membrane. This effect is experimentally calibrated and compensated during the data post processing, as explained in [11].

The capacitive sensor, built by the Waseda University in collaboration with the company Kyocera, is integrated in the CHIEF facility replacing one of the pair of windows of the test section (Fig. 2). The electrodes (in yellow) are embedded and brazed to a ceramic buffer (in white). In this way they are electrically insulated from all sensor components except from the coaxial connectors (cabled to the capacitance meter), by means of a metallic pin. The ceramic elements are inserted on the stainless steel parts (gray) which allow the mounting of the assembly on the channel assuring the tightness. A customized capacitance meter is implemented by a joint effort between the Waseda University, the JAXA and the company Baron Electric. Its measurement range is specifically tailored for the present cryogenic testing. The advantage of this meter is mainly a faster time response $(1 \mathrm{kHz})$ with respect to a commercial conditioner (normally limited to ca. $100 \mathrm{~Hz}$ ). On the contrary, the Baron output range is $0-10 \mathrm{~V}$, therefore it must be preliminary calibrated (or rely on theoretical values) to relate the electric potential and the measured capacitance.

In order to perform background light visualizations, a proper light source is positioned outside the vacuum chamber to illuminate the flow through the transparent window of the test section, just below the capacitive sensor. The camera Photron FASTCAM SA1.1 equipped with a Nikkor AF $200 \mathrm{~mm} \mathrm{f/4D} \mathrm{IF-ED} \mathrm{is} \mathrm{selected} \mathrm{to} \mathrm{record} \mathrm{the} \mathrm{movies;} \mathrm{it} \mathrm{is}$ synchronized with the acquisition of the other physical quantities. The maximum image resolution is $1024 \times 1024$ pixels at the higher acquisition speed of $5400 \mathrm{fps}$, with a minimum shutter time of $2 \mu \mathrm{s}$.

\section{Numerical results}

\subsection{Capacitive sensor accuracy}

As described in Part I [1] and in [2,12,13], the numerical results obtained with the so-called EFA, has been proved a trustful tool in order to predict the capacitive sensor performances. In this work, this methodology is again applied according to the procedure summarized in $[3,9]$ to estimate the capacitive sensor reliability in cryogenic conditions. A large variety of flow conditions are simulated with void fraction ranging from $0 \%$ to $100 \%$. Each case is considered in many different two-phase flow configurations, i.e. various bubbles distribution in the

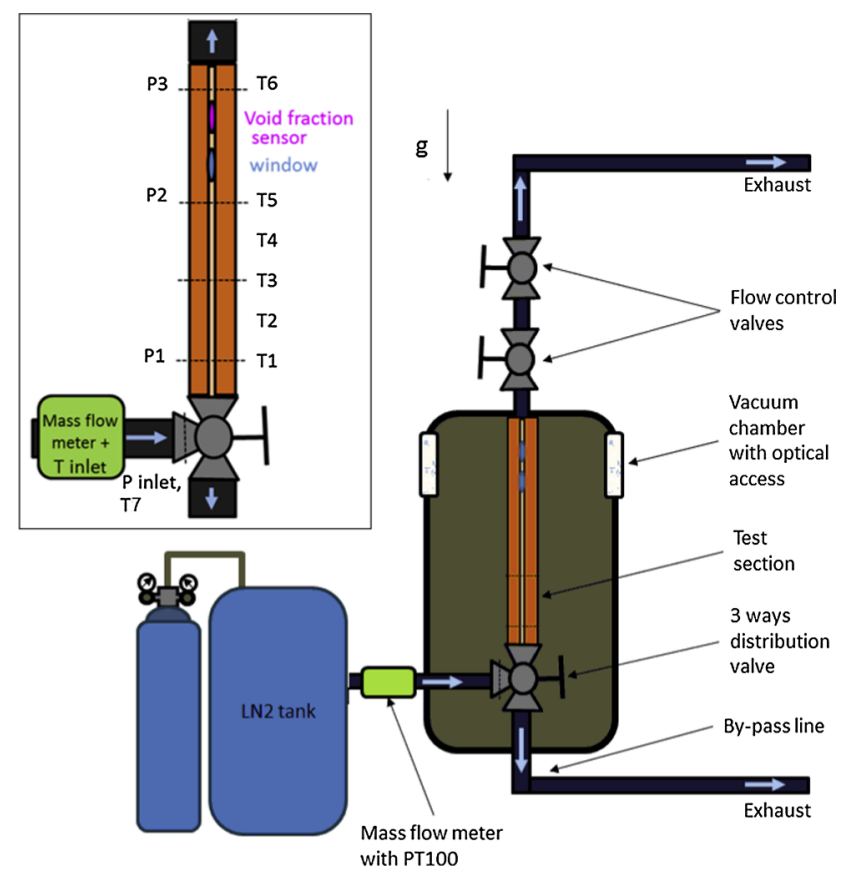

Fig. 1. Schematic of the CHIEF layout, instrument locations and nomenclature $[3,10]$. 
Table 1

Accuracy related to the measured data.

\begin{tabular}{ll}
\hline Sensor ID & Uncertainty \\
\hline$P_{1}, P_{2}, P_{3}, P_{\text {inlet }}$ & $\pm 27 \mathrm{kPa}$ \\
$\mathrm{Q}$ & $\pm 1 \% \mathrm{rdg}$ \\
$T_{1} \sim T_{7}$ & $\pm 0.5 \mathrm{~K}$ \\
$T_{\text {inlet }}$ & $\pm 2.4 \mathrm{~K}$ \\
\hline
\end{tabular}

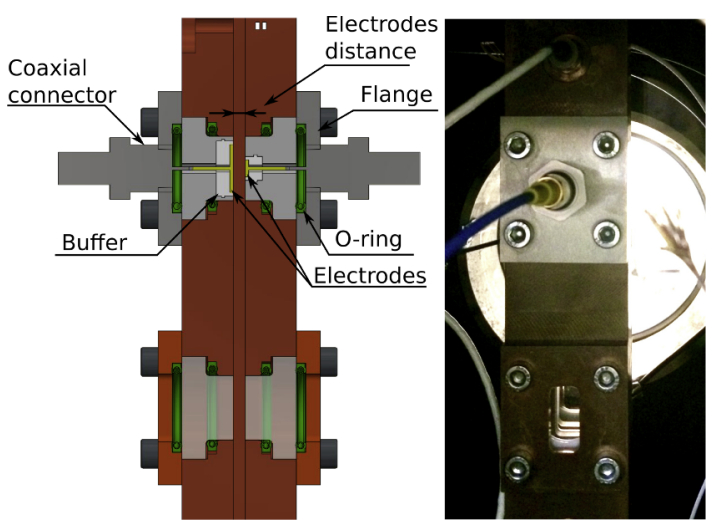

Fig. 2. Capacitive sensor integration in the CHIEF test section $[3,10]$.

measurement region, for a total of 364 EFA simulations. The analyzed cases are roughly classified in Table 2 according to the imposed void fraction (3rd column of Table 2) and its configuration in the measurement section (flow topology - 2nd column of Table 2). In the "random" conditions the flow topology simulates several types of bubbly flows, while in the "specific" cases a more structured and most probably less natural organization of the vapor and liquid phases is imposed, for example in consecutive layers. Fig. 3 provides an example of the 3D model of the sensor for one of the "random", "blockish", "annular" and "others" two-phase flow cases. The indicative distribution of the gas and liquid phases is suggested by the different colors of the area between the electrodes.

In this analysis, the dielectric constant of gas and liquid nitrogen is set respectively to 1.00973 and 1.38393 , which are the equilibrium value at a pressure of $0.4994 \mathrm{MPa}[14]$, as likely occurring during the test in the CHIEF. In Fig. 4, the EFA computations resulting capacitance and void fraction computed according to Eq. (1) [1] are provided for all the different flow configurations. The first positive remark is that the computed gas content never exceeds the imposed boundaries of $0 \%$ and $100 \%$, results that may occur in less precise sensors [2]. It can be also seen that the absolute error on the void fraction is in the $95 \%$ of the considered cases lower than $\pm 10.0 \% \mathrm{FS}$. As expected, the conditions in which the sensor appears to be less performing correspond to the "specific" cases, where very particular configurations of slug flow or artificial gas distributions are imposed. Such conditions may strongly deviate the electric field lines, decreasing the sensor accuracy. For example, when a vapor (or a liquid) pocket occupies the whole measurement volume, the medium changes exactly at the edge of the electric field creating a discontinuity where the electric lines are easier deviated (borders of the electrode). However, those kinds of two-phase flow conditions are unlikely to occur in the present study, where the regime is observed very dispersed and quite similar to the "random" cases simulated in the EFA.

Finally, the mounting and positioning of the sensor electrodes on the test section could as well play a role affecting the probe accuracy. Indeed, when the boundary conditions of single-phase gas and singlephase liquid are imposed, the capacitance obtained from the numerical results is equal to $92.93 \mathrm{fF}$ and $127.4 \mathrm{fF}$, respectively. While the values measured afterwards during the test campaign are respectively $102 \mathrm{fF}$ and $144 \mathrm{fF}$. The reason is most probably related to the electrodes relative distance: once installing and sealing them on the test section the design configuration may be slightly modified. Applying the EFA it is indeed estimated that the real electrodes distance is $0.3 \mathrm{~mm}$ lower than the design layout. However, the impact of such difference on the sensor accuracy estimation is less than $\pm 1.3 \% \mathrm{FS}$. It can be trustfully concluded that the expected absolute error on the measured nitrogen void fraction is lower than $\pm 6.8 \%$ FS with $99 \%$ C.I., when excluding the unlikely occurring "specific" flow topology (see Table 2).

\subsection{Capacitance to void fraction conversion formula}

The EFA simulations performed to estimate the capacitive sensor accuracy lead to a second fundamental result. Indeed, the linear relationship (Eq. (1) [1]), theoretically valid to translate the measured capacitance $C_{M}$ to the void fraction is improved. The modified capacitance conversion formula expressed by Eq. (2) is developed to better fit the trend of the predicted results (right side of Fig. 4).

$\alpha_{\text {linear }}=\frac{C_{L}-C_{M}}{C_{L}-C_{G}} \times 100[\%]$

$\alpha_{\text {correct }}=k \alpha_{\text {linear }}^{2}+(1-100 k) \alpha_{\text {linear }}[\%]$

The value of the coefficient $k$ depends on the distance between the probe electrodes (see Fig. 2): it is equal to 0.0015 for the design layout and to 0.0014 for the actual configuration of the sensor once installed on the test section. Assuming the design layout $(\mathrm{k}=0.0015)$, the difference between the EFA simulation results and the imposed boundary conditions, applying either Eq. (1) or Eq. (2), are compared in Fig. 5(a). When applying Eq. (2) the error decrease from $\pm 10.0 \% \mathrm{FS}$ to $\pm 9.7 \% \mathrm{FS}$ with $95 \%$ confident interval and its distribution becomes symmetric. Considering only the most realistic flow regimes ("random" cases) the error is further decreased from $\pm 6.8 \% \mathrm{FS}$ to $\pm 6.0 \% \mathrm{FS}$ with $99 \%$ confident interval. This result can be also appreciated in Fig. 5(b), which provides the comparison between the linear (Eq. (1)) and the improved relationships (Eq. (2)), fitting the EFA results previously obtained (Fig. 4). It can be seen that the modified equation decreases the mismatch between the imposed and resulting void fraction conditions. This result confirms the estimation performed during the sensor design phase [3].

Table 2

EFA simulations cryogenic two-phase flow conditions.

\begin{tabular}{lll}
\hline Case ID & Flow topology & Void fraction \\
\hline$\# 001$ & Gas & $100 \%$ \\
$\# 002$ & Liquid & $0 \%$ \\
$\# 003-\# 302$ & "Random" two-phase flow & $0-100 \%$ \\
$\# 303-\# 330$ & "Specific - Blockish" two-phase flow & $0-100 \%$ \\
$\# 331-\# 338$ & "Specific - Annular" two-phase flow & $0-100 \%$ \\
$\# 339-\# 364$ & "Specific - Others" two-phase flow & $0-100 \%$ \\
\hline
\end{tabular}

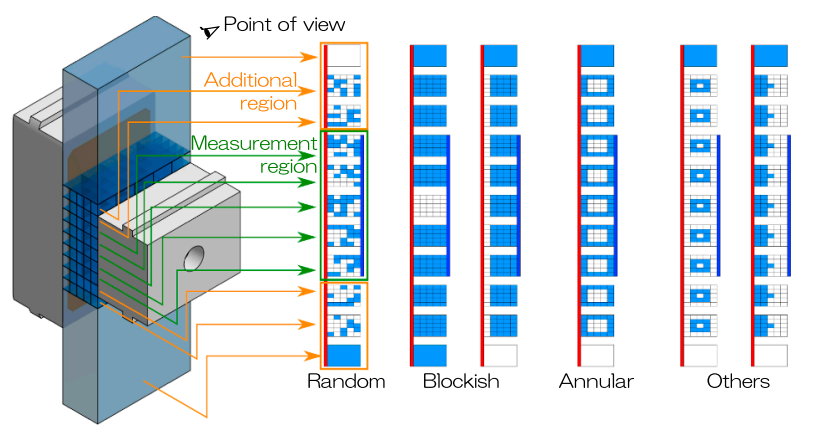

Fig. 3. Example of EFA flow configurations. 

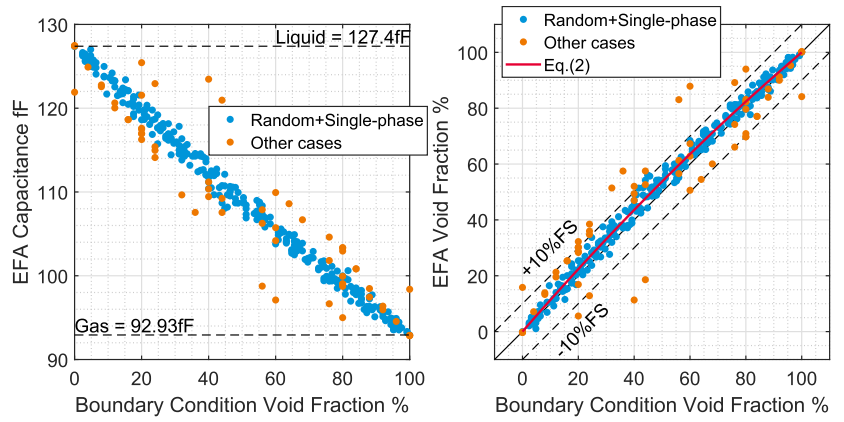

Fig. 4. EFA resulting capacitance and void fraction for 364 different imposed void fraction configurations.

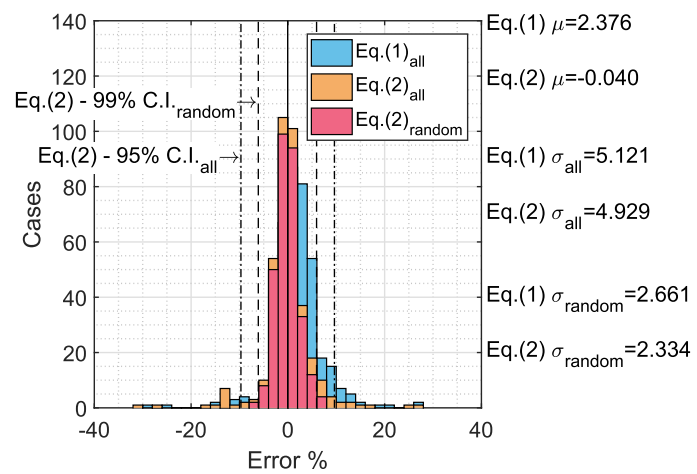

(a)

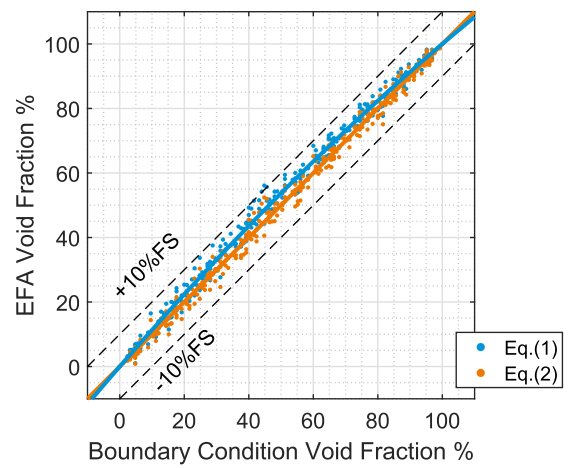

(b)

Fig. 5. EFA resulting void fraction errors distribution (a) and "random" cases results (b) using Eqs. (1) and (2).

\section{Experimental results}

Table 3 summarizes the different experimental configurations of the present campaign. It can be noted that the test matrix targets a higher mass flow rate than the nominal range for which the facility has been conceived [10]. The main reason is to ensure a sufficient cooling power to achieve a fully-liquid state at the end of the chilldown. This condition is needed for the proper validation of the capacitive sensor. Additionally, such choice allows to extend the results previously achieved

\section{Table 3}

Experimental test matrix (indicative values).

\begin{tabular}{lccccc}
\hline Test ID & $\# 1$ & $\# 2$ & $\# 3$ & $\# 3 \mathrm{~B}$ & $\# 3 \mathrm{C}$ \\
\hline $\begin{array}{l}\text { LN2 Inlet Mean } \\
\begin{array}{l}\text { Pressure [MPa] } \\
\text { LN2 Inlet Mean }\end{array}\end{array}$ & 0.6 & 0.6 & 0.6 & 0.6 & 0.6 \\
$\begin{array}{l}\text { Temperature [K] } \\
\text { LN2 Mass Flow Rate }\end{array}$ & 03 & 89 & 87 & 88 & 87 \\
$\begin{array}{l}\text { Steady State [kg/s] } \\
\text { Subcooling Method }\end{array}$ & MS & MS & HS & HS & HS \\
\hline
\end{tabular}

and to tentatively obtain a "moderate subcooling" (MS) and a "high subcooling" (HS) degree of test fluid, as detailed in [3].

\subsection{Thermal effect correction}

One of the most relevant outcomes of the cryogenic chilldown testing is the so called "thermal effect" observed on the sensor once exploited in liquid nitrogen.
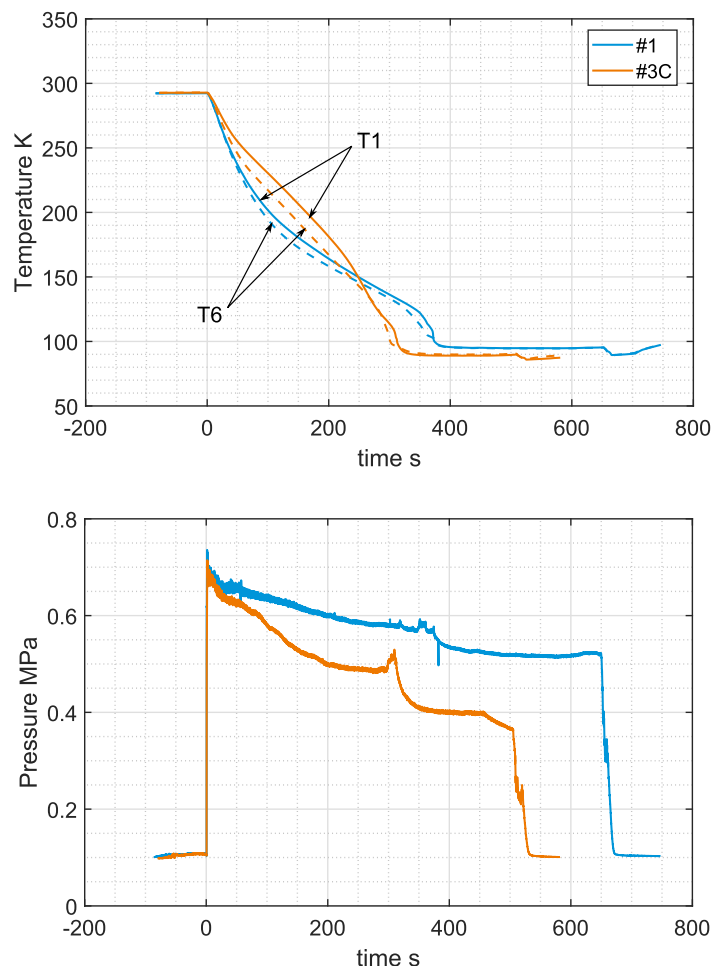

(b)

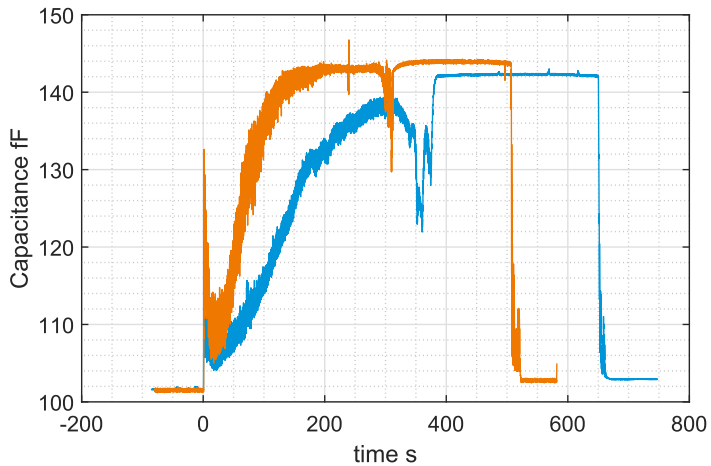

(c)

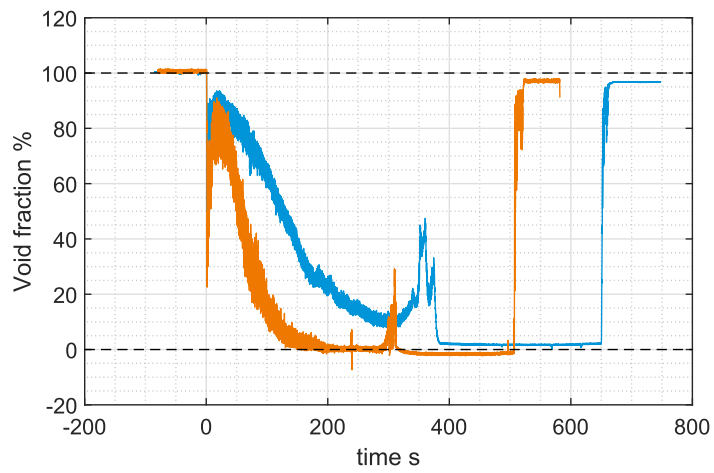

Fig. 6. "Thermal effect" appreciable comparing Test \#1 and Test \#3C - temperature $\mathrm{T} 1$ and T6 (a) pressure P3 (b) capacitance (c) void fraction (d) time histories. 
In this section, the spurious deviation of the void fraction measurements due to the cryogenic conditions is explained. A correction is proposed to properly account for low temperature. The cryogenicdriven shift in the capacitance value can be appreciated comparing two different experiments in Fig. 6 . Time $t=0 \mathrm{~s}$ corresponds to the opening of the flow distribution valve and therefore, to the beginning of the cooldown of the test section. The test is ended some minutes after the steady state is reached. Such a condition is recognized in the graph (b) by the drop of the hydraulic pressure. Considering the capacitance and void fraction values (c) and (d), it can be seen that before the start of the chilldown $(t<0 \mathrm{~s})$ the values of the two physical quantities of Test \#1 and Test \#3C are overlapped, as indeed measured at room pressure and temperature. On the contrary, once the cooling channel is at cryogenic temperature at the steady state $(400 \mathrm{~s}<t<500 \mathrm{~s})$, the measured capacitance and void fraction are different between Test \#1 and Test \#3C. In both cases, the resulting void fraction is not corresponding to the pure liquid state (i.e. $0 \%$ ) but to a slightly positive or negative value depending on the experiment condition. These measurements are not fully representative of the real physic observed by means of the pressure and temperature reached in the test section, nor in agreement with the optical visualization of the phenomena. The flow is indeed proved to be in pure liquid state at the end of the cooldown for both Test \#1 and Test \#3C [3]. The spurious effect is explained considering that the conversion formulas (Eqs. (1) and (2)) applied to retrieve the void fraction from the measured capacitance are based on the singlephase gas and single-phase liquid capacitance reference values $\left(C_{L}\right.$ and $C_{G}$ ). These values are constant at room condition while during the chilldown may vary under the influence of the flow pressure and temperature. This effect is also observed at the end of the experiments, once the flow is stopped ( $t>650 \mathrm{~s}$ for Test $\# 1$ and $t>500 \mathrm{~s}$ for Test \#3C): the pure vapor state at cold temperature is not well captured by the sensor resulting in void fraction values different from $100 \%$. Moreover, the sensor components probably shrink under the effect of the extreme cold conditions, additionally influencing the correctness of the output. It can be concluded that both the variation of the reference dielectric constants (liquid and gas phases) of the measured medium and the physical dimension of the probe are the most probable causes of the observed thermal effect.

\subsubsection{Flow permittivity variation}

The variation of the flow dielectric properties is taken into account based on the data available in literature [14]. Fig. 7 provides the trend of such characteristics for both liquid nitrogen and gas nitrogen. The gas permittivity increases when the pressure increases or when the temperature decreases. The liquid phase permittivity decreases as the pressure increases. In order to take into account the dielectric properties variation of gas and liquid nitrogen phases during the chilldown experiment, the relationships reported in Eqs. (3) and (4) are proposed. The range of validity of Eq. (3) is from $77 \mathrm{~K}$ to $350 \mathrm{~K}$ and $0.1 \mathrm{MPa}$ to 1.0 MPa. Eq. (4) is valid between 0.01 MPa and 1.47 MPa. It is assumed that the gas nitrogen has a large superheating degree. Therefore, the gas
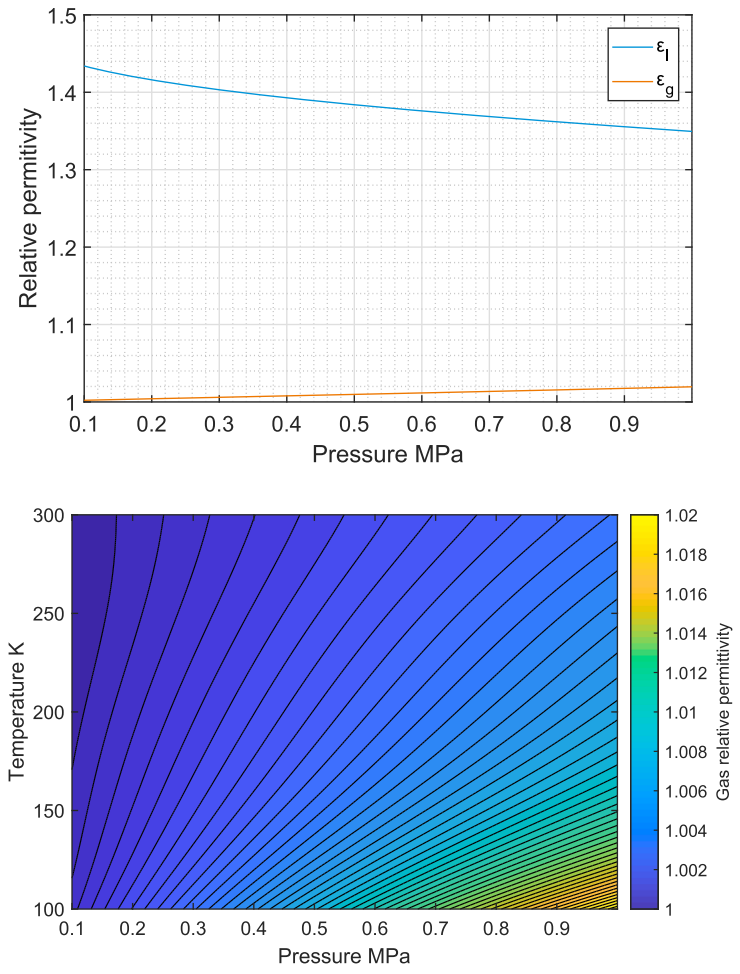

Fig. 7. Relative permittivity of gas and liquid nitrogen at saturated condition (a) and gas permittivity as function of both pressure and temperature (b).

permittivity variation is modeled as a function of both temperature and pressure. The variation of the nitrogen liquid phase dielectric property has been interpolated only as a function of the pressure. It is verified that the effect of the measured temperature on the correlation does not imply significant differences. Eqs. (3) and (4) are implemented in the data post processing to correct the reference values $C_{G}$ and $C_{L}$ according to the measured pressure and temperature during the cooldown or warm-up of the test section. Note that $[\mathrm{MPa}]$ is used as unit of pressure in the following formulas.

$$
\begin{aligned}
& \varepsilon_{G}=\varepsilon_{G}(P, T)= \\
& a_{01}+a_{02} P+a_{03} T+a_{04} P^{2}+a_{05} P T+ \\
& a_{06} T^{2}+a_{07} P^{3}+a_{08} P^{2} T+a_{09} P T^{2}+a_{10} T^{3}+ \\
& a_{11} P^{4}+a_{12} P^{3} T+a_{13} P^{2} T^{2}+a_{14} P T^{3}+ \\
& a_{15} T^{4}+a_{16} P^{5}+a_{17} P^{4} T+a_{18} P^{3} T^{2}+ \\
& a_{19} P^{2} T^{3}+a_{20} P T^{4}+a_{21} T^{5} \\
& \varepsilon_{L}=\varepsilon_{L}(P)= \\
& b_{01}+b_{02} P+b_{03} P^{2}+b_{04} P^{3}+b_{05} P^{4}+b_{06} P^{5}
\end{aligned}
$$

Where:

$$
\begin{aligned}
& a_{01}=1.004 \\
& a_{02}=-1.409 \times 10^{-4} \\
& a_{03}=6.353 \times 10^{-2} \\
& a_{04}=1.690 \times 10^{-6} \\
& a_{05}=-8.940 \times 10^{-4} \\
& a_{06}=2.029 \times 10^{-2} \\
& a_{07}=-9.439 \times 10^{-9} \\
& b_{01}=1.470 \\
& b_{02}=-4.348 \times 10^{-1}
\end{aligned}
$$

$$
\begin{aligned}
& a_{08}=5.703 \times 10^{-6} \\
& a_{09}=-2.835 \times 10^{-4} \\
& a_{10}=3.903 \times 10^{-3} \\
& a_{11}=2.476 \times 10^{-11} \\
& a_{12}=-1.669 \times 10^{-8} \\
& a_{13}=1.221 \times 10^{-6} \\
& a_{14}=-1.209 \times 10^{-5} \\
& b_{03}=1.039 \\
& b_{04}=-1.425
\end{aligned}
$$

$$
\begin{aligned}
& a_{15}=-2.474 \times 10^{-3} \\
& a_{16}=-2.457 \times 10^{-14} \\
& a_{17}=1.810 \times 10^{-11} \\
& a_{18}=-1.648 \times 10^{-9} \\
& a_{19}=8.714 \times 10^{-9} \\
& a_{20}=9.553 \times 10^{-7} \\
& a_{21}=1.012 \times 10^{-3} \\
& b_{05}=9.208 \times 10^{-1} \\
& b_{06}=-2.225 \times 10^{-1}
\end{aligned}
$$




\subsubsection{Capacitive sensors thermal contraction}

In order to collect the necessary information of the capacitance change related to the cooldown of the sensor, the "warm-up" data are exploited: the capacitive sensor output is recorded during the warming of the test section, after the end of the chilldown experiments. In such conditions, the pressure inside the cooling channel is at ambient value $\left(P_{G, \text { ref }}=0.1 \mathrm{MPa}\right)$, while the temperature rises up to room temperature $\left(T_{G, \text { ref }}=273 \mathrm{~K}\right)$. In addition to the effect of the thermal contraction/ expansion of the sensor, the "warm-up" recording includes the effect detailed in the previews section: the contribution related to the dielectric property changes. The cold gas left in the test section is indeed warming up together with the probe and the rest of the facility components. Fig. 8 shows the experimental values measured during the warm-up normalized to the capacitance reference value at $273 \mathrm{~K}$ and ambient pressure. It can be seen that the effect related to the gas permittivity variation (expressed by Eq. (5)) is therefore subtracted to the experimental points to define a corrective equation only related to the shrinking of the sensor parts in the cold environment (Eq. (6)). The resulting thermal contraction (TC) fitting $f_{T C}$ is reported in Eq. (7).

$\left.\frac{C(T)}{C_{r e f}}\right|_{\varepsilon_{G}}=\frac{\varepsilon_{G}\left(P_{G, r e f}, T\right)}{\varepsilon_{G}\left(P_{G, r e f}, T_{G, r e f}\right)}$

where $\varepsilon_{G}$ is defined by Eq. (3).

$\left.\frac{C(T)}{C_{r e f}}\right|_{T C}=f_{T C}(T)$

With:

$f_{T C}=c_{01}+c_{02} T+c_{03} T^{2}+c_{04} T^{3}+c_{05} T^{4}+$

$c_{06} T^{5}+c_{07} T^{6}+c_{08} T^{7}+c_{09} T^{8}+c_{10} T^{9}$

Where:

$$
\begin{array}{ll}
c_{01}=-5.56092 \times 10^{-19} & c_{06}=8.88606 \times 10^{-7} \\
c_{02}=7.41453 \times 10^{-16} & c_{07}=4.84551 \times 10^{-5} \\
c_{03}=-4.01971 \times 10^{-13} & c_{08}=-8.46797 \times 10^{-3} \\
c_{04}=1.11873 \times 10^{-10} & c_{09}=1.31014 \\
c_{05}=-1.61248 \times 10^{-8} & c_{10}=1.30590
\end{array}
$$

\subsubsection{Thermal effect corrected results}

In Eqs. (8) and (9), the capacitance values of liquid and gas nitrogen are corrected according to the physical properties variations within the pressure and temperature working ranges (Eqs. (3) and (4)) and to the thermal contraction of the sensor parts (Eq. (7)). These equations are then coupled to Eqs. (1) and (2) to retrieve the void fraction during the chilldown experiments.

$$
\begin{aligned}
& C_{G}(P, T)=C_{G, S} \times \frac{f_{T C}(T)}{f_{T C}\left(T_{G, S}\right)} \times \frac{\varepsilon_{G}(P, T)}{\varepsilon_{G}\left(P_{G, S}, T_{G, S}\right)} \\
& C_{L}(P, T)=C_{L, S} \times \frac{f_{T C}(T)}{f_{T C}\left(T_{L, S}\right)} \times \frac{\varepsilon_{L}(P)}{\varepsilon_{L}\left(P_{L, S}\right)}
\end{aligned}
$$

where:

$$
\begin{array}{lll}
C_{L, S}=143.1 \mathrm{fF} & P_{L, S}=0.46 \mathrm{MPa} & T_{L, S}=92.51 \mathrm{~K} \\
C_{G, S}=101.7 \mathrm{fF} & P_{G, S}=0.10 \mathrm{MPa} & T_{G, S}=273.0 \mathrm{~K} .
\end{array}
$$

Fig. 9 proposes the comparison between the measured void fraction, before (a) and after (b) the aforementioned data corrections, for the Test \#1 and Test \#3C. It can be noticed that when the compensation is applied, at the end of chilldown both the experiments reach the pure liquid state ( $0 \%$ void fraction) within a maximum difference of $\pm 2 \% \mathrm{FS}$, independently on the temperature and pressure at the steady state. Moreover, once the mass flow rate is stopped, the void content detected by sensor, applying the described corrections, is indeed $100 \%$, within $\pm 1 \%$ FS error, even if the gas in the measurement section is at very cold temperature. These results show that the single-phase deviations are successfully decreased, and that the compensation is well correcting the spurious effects due to the cryogenic conditions. The thermal effect influence on the measured void fraction is now reduced from more than $\pm 5 \% \mathrm{FS}$ to less than $\pm 2 \% \mathrm{FS}$.

Nevertheless, it should be underlined that the thermal effect corrections are most probably less effective when the two-phase flow is highly unsteady such as before the Leidenfrost point (transitional regime). It can be observed that in such region the resulting void fraction may be not very precisely captured, even if the thermal corrections are applied. It is assumed that probably the thermal contraction model is less applicable in those conditions because it is developed on the warmup quasi-stable data (Eq. (7)). On the contrary, during the chilldown the test section wall next to the capacitive sensor and the sensor itself may be characterized by slightly different temperatures because of the very fast thermal behavior of chilldown. A possible improvement of the drift correction would be, therefore, obtained if the different temperatures of the sensor parts are precisely known. Additionally, the flow temperature is considered equal to the wall temperature in both Eqs. (3) and (4). Not measuring the exact fluid temperature but only the channel wall temperature, it can be anticipated that this effect could not be better modeled to take into account the subcooled degree of most of the experiments. It is reminded, indeed, that the present test campaigns consider a range of mass flow rate and a subcooling degree different than the usual CHIEF working conditions in almost all the experiments. In such experimental cases it cannot be excluded that the measured wall temperature may less reliably represents the actual fluid temperature (faster cooling phenomena and colder fluid bulk temperature). A wider parametric study and possibly a reference metrology would be required to conclude more about the capacitive sensor performance measuring the void fraction content in the complex boiling regime occurring before the Leidenfrost point oscillation.

\subsection{Void fraction to light intensity correlation}

The high-speed camera images acquired during the test section chilldown are post processed and compared to the void fraction measurements. During the experiments performed for the isothermal validation of the sensor (Part I of the present work [1]), the flow topology was predominantly bubbly flow. On the contrary, the chilldown phenomenon involves very complex flow regimes: depending on the cooldown phase, gas pockets are followed by a very dense bubbly flow, becoming then annular/dense bubbly, slug/bubbly and finally bubbly, before the single-phase convection heat transfer mode is achieved (Fig. 10). Therefore, the high-speed imaging could not be exploited as reference metrology as done during the bubble column testing (Part I [1]), but an attempt is done to correlate the light intensity to the capacitive data. It appears, indeed, that the flow regimes captured in the videos could be directly linked to the gas content retrieved by the sensor. In Fig. 10 each high speed image is shown in its first half as

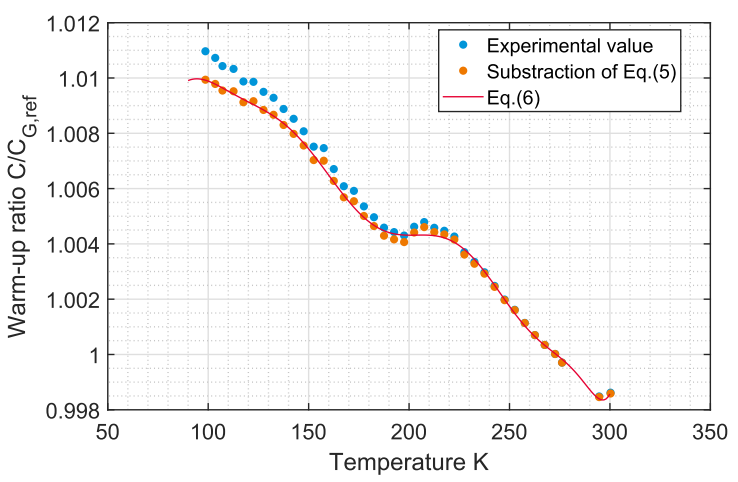

Fig. 8. Relative capacitance variation during test section warm-up. 


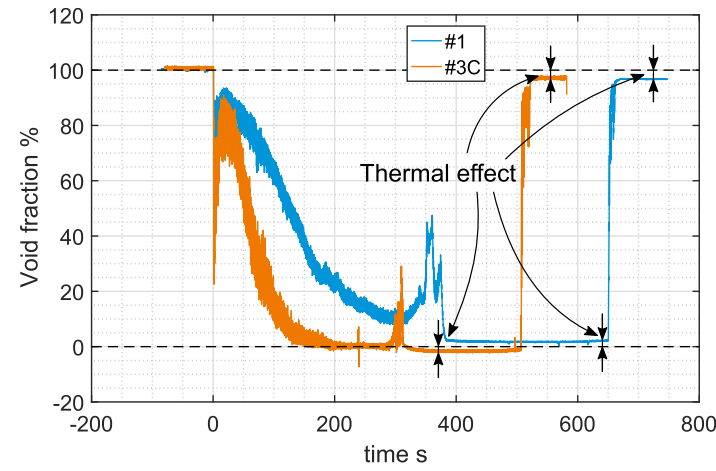

(a)

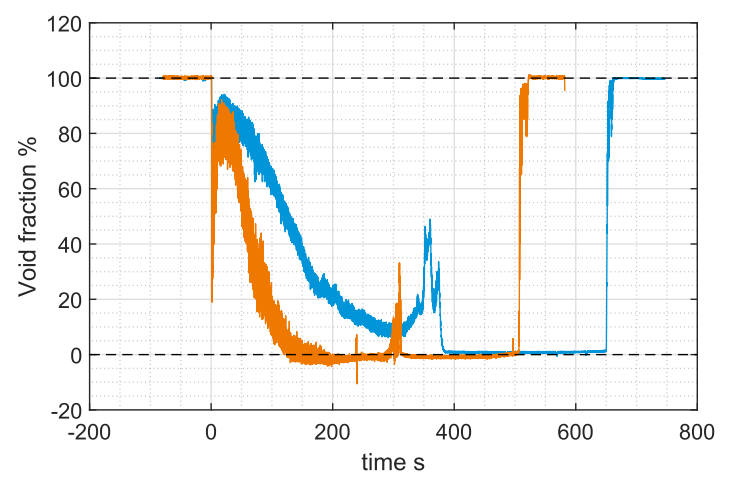

(b)

Fig. 9. Test \#1 and Test \#3C void fraction time histories before (a) and after (b) the "thermal effect" correction.

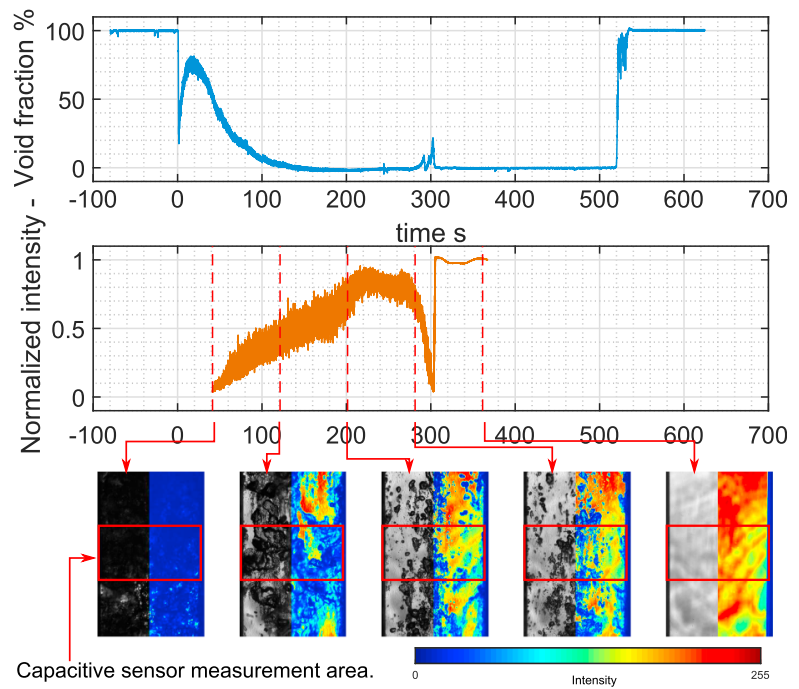

Fig. 10. Qualitative correlation between the measured void fraction and the high-speed images light intensity.

originally acquired and in the second half post processed to enlighten the light intensity. The light intensity is computed in the area of the images equivalent to the probe measurement volume (red area in Fig. 10 frames). It may vary from 0 (black level) to 255 (white level). For each frame, the mean light intensity in the mentioned area is computed averaging all the pixel values. The result is normalized by the background intensity. The output intensity varies now between zero and one.

Fig. 11 represents the obtained light intensity as function of the void fraction for all the experiments. The pure gas region and the pure liquid region are characterized by a light intensity equal to one: the light well reaches in both cases the camera CCD. The dense bubbly two-phase flow is represented by lower gray scales, between 0 and 0.5 : depending

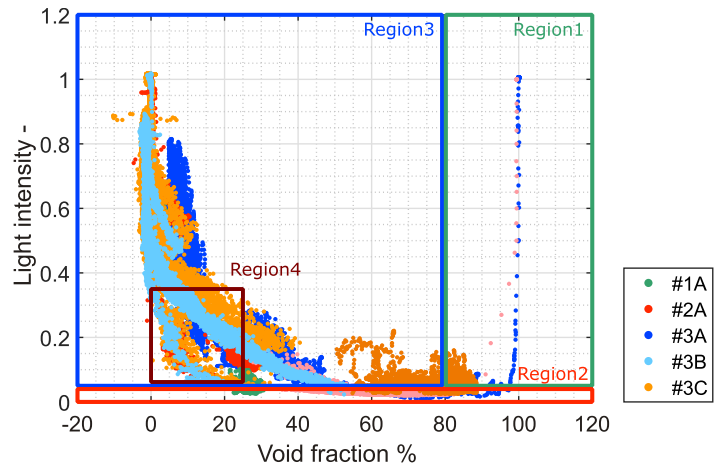

Fig. 11. High-speed camera images light intensity correlated to the measured void fraction for all the experiments.

by the amount of bubbles the background light may be dramatically scattered and deviated, resulting in a dark image. Roughly, four different flow regimes could be identified based on the void fraction content and the light intensity (L. I.):

Region 1: High void fraction regime: L. I. $>0.04$ and $\alpha>80 \%$

Region 2: Opaque two phase flow regime: L. I. $\leq 0.04$

Region 3: Heterogeneous regime: L. I. $>0.04$ and $\alpha \leq 80 \%$

Region 4: Near Leidenfrost region: L. I. $\leq 0.35$ and $\alpha \leq 25 \%$

Regions 1 and 2 are very difficult to characterize for the current purpose: the phenomena is very unsteady in Region 1 while in Region 2 the light intensity is not enough to allow a proper determination of the flow topology. Both such issues are encountered near the Leidenfrost point. Therefore, Region 4 is not suitable for the current intent. The present analysis focuses on Region 3. The obtained void fraction/light intensity correlation is expressed by Eq. (10).

$\alpha=68.72 \times e^{-6.522 L . I .}$

Eq. (10) is plotted in Fig. 12 as the solid line, within a 99\% confident interval range (dashed lines). The results show that the capacitancebased void fraction, in Region 3, can be correlated to the high-speed visualizations through the light intensity according to Eq. (10) within a $\pm 10 \% \mathrm{FS}$ discrepancy.

Finally, the comparison between the void fraction computed by means of the light intensity and capacitance-based void fraction is provided in Fig. 13. It can be seen that the discrepancy between both methods are at the most ca. $20 \%$ in absolute void fraction for Region 3. While for the other flow regimes, as expected, a less good agreement is found. For example, as shown in Fig. 13, the capacitive sensor is more reactive to the void fraction oscillations at the Leidenfrost point: two vaporization peaks are detected in Test \#2, \#3B and \#3C, which are not captured by the optical method.

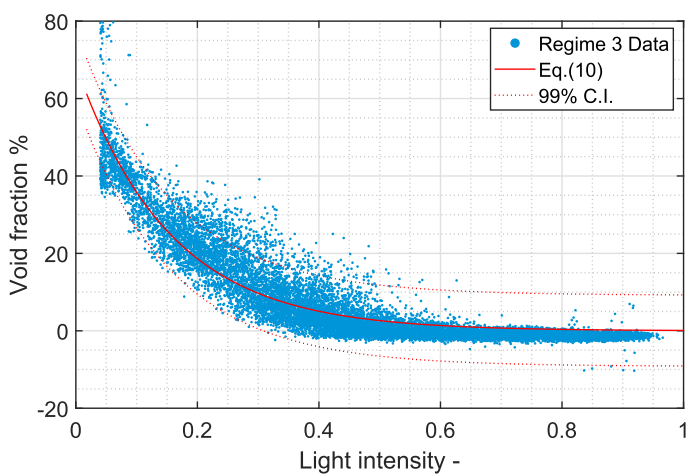

Fig. 12. Measured void fraction correlated to the high-speed camera images light intensity for all the experiments, Regime 3. 

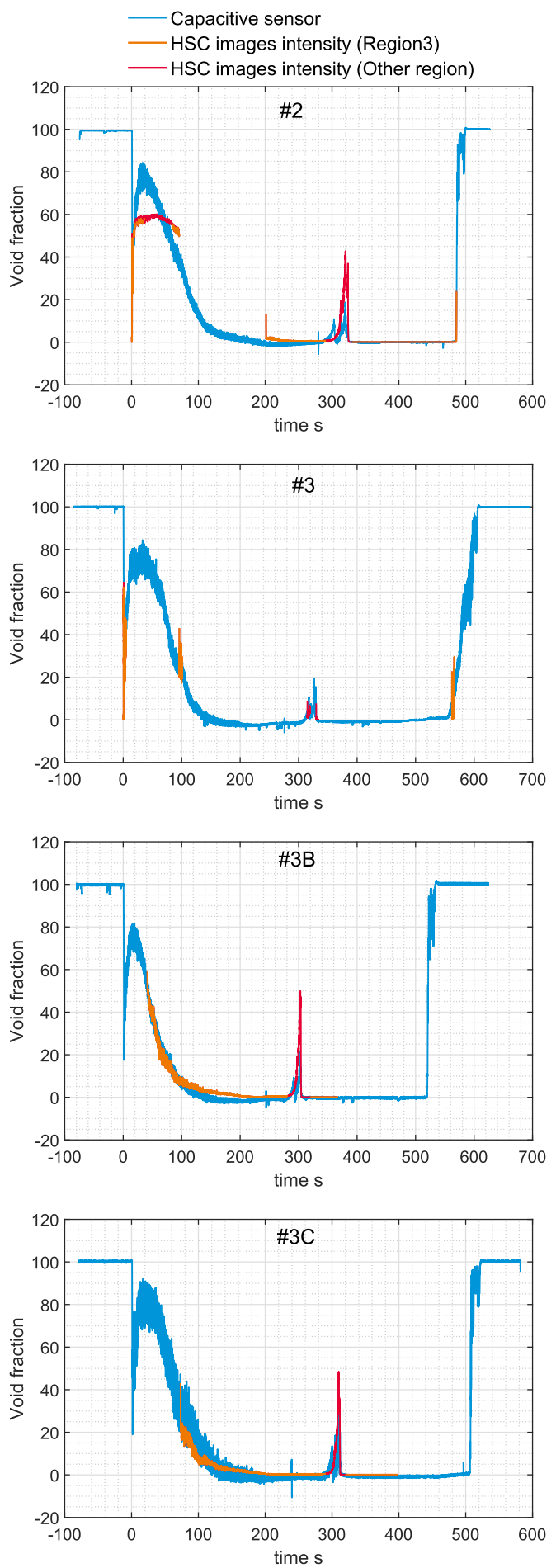

Fig. 13. Comparison between the void fractions computed with Eq. (10) and the capacitive sensor for some of the experiments.

\subsection{Capacitive sensor time response}

By means of the high-speed camera recordings, the time response of the capacitive sensor has been also assessed. Such performance strongly depends on the capacitance meter. The Baron circuit time response is equal to $32 \mathrm{kHz}$. It is equipped by a $3.2 \mathrm{kHz}$ cutoff filter, therefore, at least $1 \mathrm{kHz}$ frequency response is expected for the void fraction measurements. In Fig. 14, the response time of the sensor is evaluated comparing the capacitive signal and high-speed camera acquisitions. It can be seen that even if the $1 \mathrm{kHz}$ void fraction signal from the
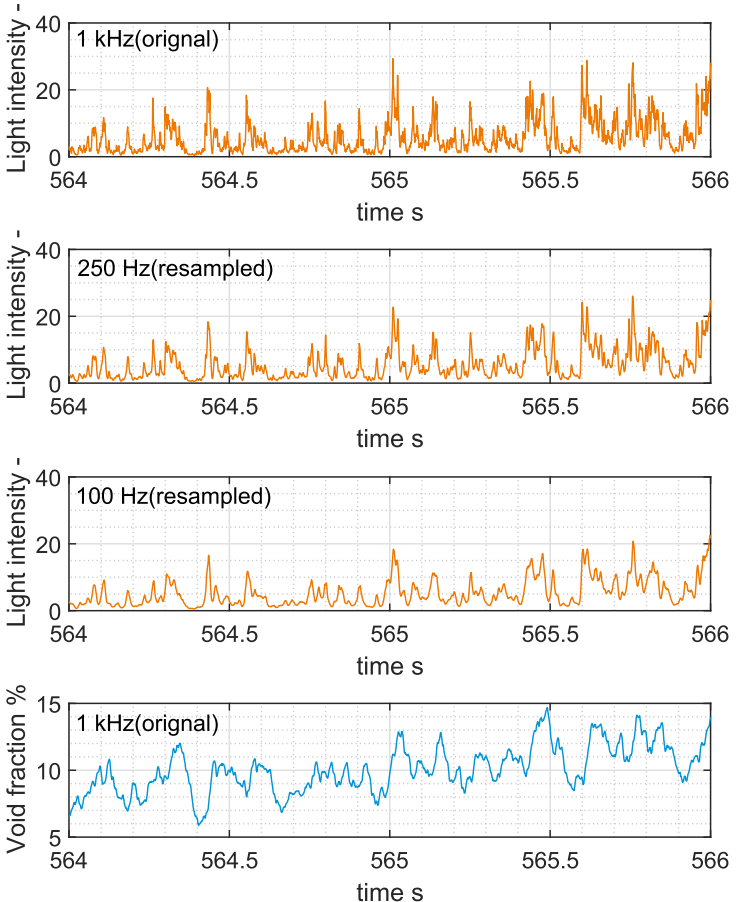

Fig. 14. Comparison between the high-speed images light intensity and the capacitive sensor time response (Test \#3).

capacitive sensor is capable to detect most of the relevant fluctuations at the correct time instant, the data appears to be smoother than the movies recordings. In particular, the $100 \mathrm{~Hz}$ light intensity signal obtained by the visualizations seems to represent at best the capacitancebased void fraction.

The Fast Fourier Transformations of the void fraction time evolution
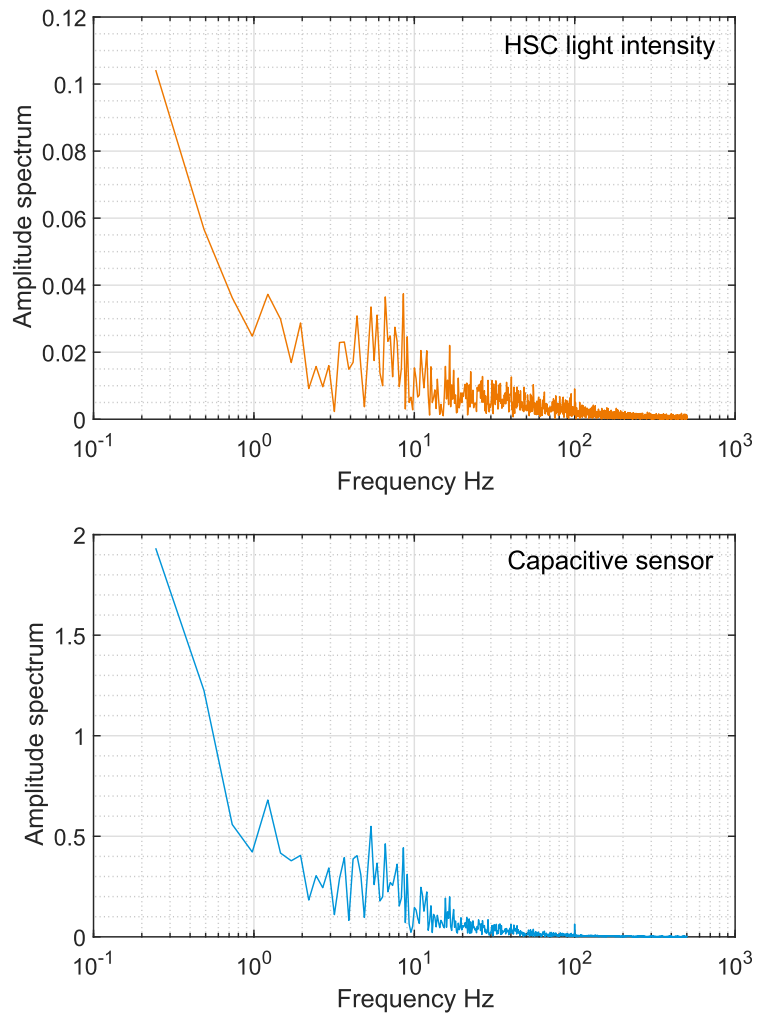

Fig. 15. Comparison between the high-speed images light intensity and the capacitive sensor FFT (Test \#3). 
obtained from the light intensity and from the capacitive sensor are compared in Fig. 15. The results show that the most of the high frequency components (higher than $100 \mathrm{~Hz}$ ) can be captured only by the optical technique. In the current experiment, it was not possible to asses if the high frequency fluctuations measured by means of the high-speed camera would be actually related to the chilldown phenomenon or to spurious noise (for example, the background light oscillation). Thus, since void fraction fluctuations higher than $100 \mathrm{~Hz}$ could not be observed, it can be concluded that the capacitive sensor time response is at most $100 \mathrm{~Hz}$.

\section{Conclusion}

In the present work, the development and the evaluation of the performances of a void fraction sensor capable of detecting the gas content during the liquid nitrogen chilldown phenomenon has been presented. In particular, the EFA is extensively applied to simulate the cryogenic two-phase flow; it allows the improvement of the linear relationship between the probe capacitance and the void fraction (Eqs. (1) and (2)). The expected accuracy on the measured nitrogen void fraction is then assessed to be lower than $\pm 6.0 \% \mathrm{FS}$ within a $99 \%$ confidence level. Concerning the experimental outcomes, the observed "thermal effect" on the measured void fraction is modeled and compensated: its impact on the results is decreased from more than $\pm 5 \% \mathrm{FS}$ to less than $\pm 2 \%$ FS. Such achievement could not be verified in twophase flow but only in single-phase conditions by means of high-speed camera background lighting visualizations as well as the recorded pressure and temperature boundary conditions. In transitional flow regimes (such as near the Leidenfrost point), the proposed correction less effectively applies. The optical technique is exploited as additional metrology supporting the understanding of the results. Then, the capacitance-based void fraction is successfully correlated to the images light intensity within about $\pm 20 \%$ discrepancy for the specific flow topology here defined as Heterogeneous regime. Finally, it is concluded that the time response of the capacitive sensor is at the most $100 \mathrm{~Hz}$.

\section{Acknowledgement}

The authors gratefully thank the financial support of this study by the ESA GTSP contract N 4000111618/14/NL/PA. Additionally, the EM EASED program which allowed Mr. Sakamoto permanence at the VKI during the accomplishment of this work. We also acknowledge the Baron Electronic and Kyocera companies for the manufacturing of the capacitance measurement circuit and probe.

\section{References}

[1] Sakamoto Yuki, Peveroni Laura, Kobayashi Hiroaki, Sato Tetsuya, Steelant Johan, Vetrano Maria Rosaria. Void fraction measurement in cryogenic flows. Part I: Design and validation of a void fraction capacitive sensor. Cryogenics 001122752018;94:36-44. https://doi.org/10.1016/j.cryogenics.2018.07.004.

[2] Sakamoto Y, Sato T, Kobayashi H. Development study of a capacitance void fraction sensor using asymmetrical electrode plates. J Fluid Sci Technol 2016;11(2):JFST0008. https://doi.org/10.1299/jfst.2016jfst0008.

[3] Peveroni L, Sakamoto Y, Fouchier C, Steelant J, Buchlin J-M. Liquid nitrogen chilldown: void fraction measurements and feasibility of extension to liquid methane testing. In: Submitted to the space propulsion conference 2018, Seville, Spain; 2018.

[4] Hinata S, Venart J, Sousa A, Sakurai M, Nakazawa M, Ichimura I. Miniature optical fiber sensor used to measure the local void fraction. Trans Jpn Soc Mech Eng Ser B 1990;56(525):1433-9. https://doi.org/10.1299/kikaib.56.1433.

[5] Vieira RE, Kesana NR, Torres CF, McLaury BS, Shirazi SA, Schleicher E, et al. Experimental investigation of horizontal gas-liquid stratified and annular flow using wire-mesh sensor. J Fluids Eng 2014;136(12):121301. https://doi.org/10. 1115/1.4027799.

[6] Rocha MS, Simões-Moreira JR. Void fraction measurement and signal analysis from multiple-electrode impedance sensors. Heat Transfer Eng 2008;29(11):924-35. https://doi.org/10.1080/01457630802186015.

[7] Hori K, Fujimoto T, Kawanishi K, Nishikawa H. Development of an ultra-fast X-ray computed tomography scanner system. Trans Jpn Soc Mech Eng Ser B 1998;64(617):239-44.

[8] Murai Y, Ohta S, Shigetomi A, Tasaka Y, Takeda Y. Development of an ultrasonic void fraction profiler. Meas Sci Technol 2009;20(11):114003. https://doi.org/10. 1088/0957-0233/20/11/114003.

[9] Sakamoto Y, Peveroni L, Kobayash H, Minote K, Tane S, Sato T, et al. Development study of capacitive void fraction sensor for cryogenic chilldown experiment. In: Proceedings of the JSRAE annual conference; 2017 [In Japanese].

[10] Peveroni L, Steelant J, Vetrano MR. Experimental investigation of liquid nitrogen chilldown two phase flow. In: Space propulsion 2016; 2016.

[11] Peveroni L, Pinho J, Vetrano MR, Strengnart M, D’Agruma J. Gstp4 multiphysics valve modelling, TN4100 - test report. Tech Rep; 2014.

[12] Sakamoto Y, Sato T, Kitakoga S, Sumi Y, Ebisuno S, Uragaki K, et al. Development study of a capacitance void fraction meter using asymmetric electrode plates. In: Proceedings of the 55th conference on aerospace propulsion and power; 2015 [In Japanese].

[13] Sakamoto Y, Sato T, Kobayashi H, Uragaki K, Tane S, Minote K. Development study of a capacitance based void fraction sensor for cryogenic two-phase flow. In: AJCPP2016; 2016.

[14] Younglove BA. Erratum: Thermophysical properties of fluids. i. argon, ethylene, parahydrogen, nitrogen, nitrogen trifluoride, and oxygen. J Phys Chem Ref Data 1985;14(2):619. https://doi.org/10.1063/1.555731. 